\section{Travel-Mode Detection Based on GNSS Trajec tories and Heart-Rate Data from Fitness Watches}

GI_Forum 2018, Issue 2 Page: 261 - 274 Full Paper

Corresponding Author: petra.stutz@sbg.ac.at DOI: 10.1553/giscience2018_02_s261

\author{
Petra Stutz and Eva Westermeier \\ University of Sa lzburg, Austria
}

\begin{abstract}
With the advent of wearable GNSS devices and activity trackers, new opportunities for automatic travel-mode detection arise. Although physiological measures such as heart rates camy a high potential for travel-mode detection, little research has been done that exploits this data. This paper presents a rule-based method for the detection of the travel modes walk, bike, bus, tra in and car, based on the combination of GNSS and heart-rate data from off-the-shelf fitness watches. The aim of this research is to minimize the input variables and reference data for mode detection. In the case study, the proposed workflow performed very well and substantially reduced the confusion between active and motorized travel modes compared to a workflow that did not take heart rate into consideration, although the differentiation among motorized travel modes could be further enhanced with additional data. Combining GNSS data with physiological variables such as heart rate allows a clear reduction in the amount of reference data and processing effort required for mode detection.
\end{abstract}

\title{
Keywords:
}

tra vel-mode detection, active mobility, trip segmentation, physiologic al data, GPS

\section{Introduction}

Understanding people's everyday mobility with respect to different modes of transport is crucial for fields such as transport modelling and planning. While traditional methods focus on questionnaires and mobility diaries, in recent decades more studies have made use of technological approaches and analysed volunteers' everyday-mobility movement data, recorded using wearable GNSS (Global Navigation Satellite System) loggers and accelerometers (Nitsche, Widhalm, Breuss, Brändle \& Maurer, 2014; James et al. 2016; SiłaNowicka et al., 2016). Movement data from GNSS loggers consist of a temporal sequence of recorded point locations, which we refer to as records. One trajectory, that is one countable journey from start to end point, can consist of multiple modes, for example going by bike to the station, then going a few stops by train, and afterwards walking from the station to the final destination. These intermodal trips are of great interest to mobility research. Travelmode detection (TMD) aims to divide trajectories into single-mode segments and identify the respective modes. 
In addition to GNSS data, the spread of wearable location-aware activity trackers enables inexpensive collection of heart rate and other physiological parameters. Heart rates exhibit a relatively clear relationship, across different types of physical activity of moderate to high intensity, with the intensity of the activity (Brage et al., 2007). The potential of heart rates for travel-mode detection, especially for the distinction between active and motorized movement, is thus obvious. Although used for related purposes (e.g. Costa et al., 2015), to the best of our knowledge no studies have successfully used the combination of GNSS and heart-rate data for travel-mode detection.

In this paper, we propose a rule-based method for travel-mode detection based on data from off-the-shelf fitness watches. Our method makes use of the additional category of physiological indicators (heart rate) and relies exclusively on GNSS and heart-rate data. This could help distinguish active and motorized travel modes more efficiently, and substantially reduce the number of input variables and reference data required for travel-mode detection.

The remainder of this paper is structured as follows: Section 2 gives an overview on related work; Section 3 describes the proposed method for travel-mode detection; Section 4 presents a case study where the method was successfully applied. In Section 5, the results are summarized and discussed along with potential starting points for further research.

\section{Related Work}

Existing methods on travel-mode detection often rely on map-matching, where trajectories are matched to a reference graph in order to identify the road that a vehicle or person was moving along (Quddus, Ochieng \& Noland, 2007). The underlying part of the road graph can then give information about the travel mode - e.g. a vehicle travelling along a bus lane is most probably a bus. One of the drawbacks of map-matching is its dependence on a highly accurate road graph. In addition, map-matching is particularly difficult for bike trips, e.g. due to narrow bike lanes next to roads and the variability of cyclists' behaviour.

While most previous studies on travel-mode detection use machine learning or probabilistic methods, a range of researchers also use rule-based methods (e.g. Bohte \& Maat, 2009; Gong, Chen, Bialostozky \& Lawson, 2012; Stopher et al., 2008). Gong, Morikawa, Yamamoto \& Sato (2014) identify three categories of input variables to determine travel modes: GNSS-related indicators such as speed and acceleration; GIS indicators such as road network, rail lines or bus stops; the information provided by study participants. Zong et al. (2017) provide a table of characteristic parameter values which can serve as a reference for ruleset development.

Physiological indicators such as heart rate represent an additional category of indicators for travel-mode detection. Existing research on heart rates in different travel modes mostly focuses on the time spent moving in a particular mode in order to quantify health impacts of commuting (Ogilvie et al., 2016; Costa et al., 2015). In a study that went beyond travel-mode detection, Pärkkä et al. (2006) used over 20 different sensors, including GPS and physiological sensors, for the classification of everyday activities. In their study setup, heart rates did not represent a helpful indicator for activity recognition due to high inter-individual 
differences. It should be noted that the relationship between heart rate and physical activity intensity varies significantly between individuals, depending on factors such as gender, age and fitness. For a meaningful comparison of heart rates, it is thus necessary to calibrate them. As Brage et al. (2007) and others have shown, the simple approach of using heart rates above resting level while adjusting for gender can already make heart rates more comparable. For instance, Costa et al. (2015) calibrated their heart-rate data based on sleeping heart rates, age and gender. However, in anonymized data this individual-based information is not available and an alternative calibration approach is required. Our approach addresses these issues as it leverages anonymized heart-rate data using an inexpensive calibration, and thus helps to overcome the need for prior map-matching.

\section{Methods}

The method proposed here relies on GNSS and heart-rate data from off-the-shelf fitness watches, with a regular high sampling interval (i.e. a few seconds) for both GNSS location and heart rate. This approach allows the number of input variables to be minimized, and dispenses with the need for prior map-matching as well as other indicators, such as acceleration and heading. Heart rates are made comparable using a calibration based on an approximated resting heart rate, thus making the method suitable for anonymized data.

After data preprocessing (Section below), trajectories are segmented in a two-stage procedure (Section 'Two-stage Trajectory Segmentation'). The travel modes are classified following a specified hierarchy, based on iterative ruleset adjustment (Section 'Rule-based Travel-Mode Detection - TMD' + see Figure 1). The applicability of the method is proved in Section 'Case Study'.

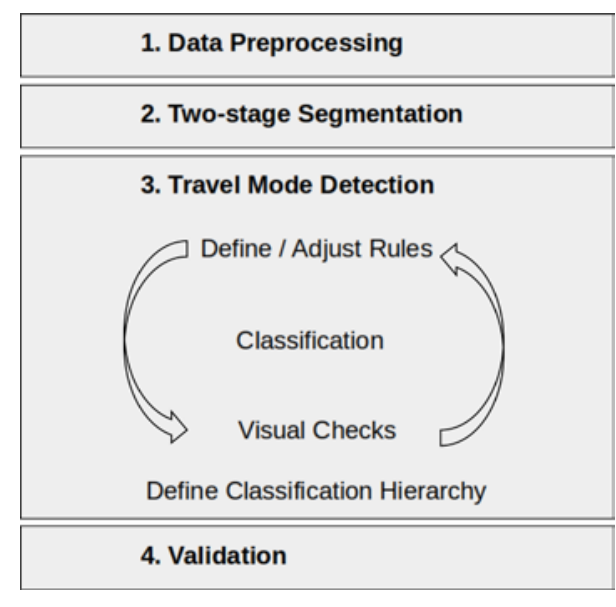

Figure 1: Workflow 


\section{Data Preprocessing}

Fitness watches commonly allow the recording of individual trajectories, where the user actively has to start and stop the recording. Leveraging the regular sampling interval, the speed between each position recorded can be calculated using the distance from the previous record. As off-the-shelf fitness watches do not usually provide GNSS accuracy indicators such as dilution of precision (DOP), records with low locational accuracy cannot be filtered in advance and become evident as zero or unrealistically high speed values. As speed values are a major indicator for trajectory segmentation and travel-mode detection in our proposed method, it is necessary to systematically filter out speed outliers and correct respective speed values. More details on the correction of speed values as implemented in the case study are provided in Section 4.2.

\section{Two-stage Trajectory Segmentation}

To divide trajectories into single-mode segments, this approach uses a two-stage segmentation process inspired by Zhang, Dalyot, Eggert \& Sester (2011). First, trajectories are segmented according to stops, while in the second stage trajectories are divided into single-mode segments.

\section{Stop-Point Detection and Segmentation}

Assuming that mode changes can only occur at stop points, these must first be identified. Stop points occur when a person's or a vehicle's movement comes to a halt, for instance in stop-and-go traffic, at bus stops or at traffic lights. To account for measurement errors, the average speed of two consecutive GNSS records is used. As suggested by Gong et al. (2012), a threshold of $1.6 \mathrm{~km} / \mathrm{h}$ can be used for the initial detection. In the second step, records below $3.6 \mathrm{~km} / \mathrm{h}$ within five metres of the initial stop points are also classified as stop points. Trajectories are then divided into segments based on stop points, which we refer to as stoppoint segments. Enabled by the high sampling frequency, this method offers a fine-grained detection of stop points and stop-point segments. To avoid a high number of very short, irrelevant segments, stop-point segments below a specified number of records can also be declared stop points. The duration, length and speed-related indicators are then derived from each stop-point segment.

\section{Mode-Change Detection and Identification of Single-Mode Segments}

Up to this point, trajectories are divided into segments based on stops, but there is no information regarding where the travel mode was changed. To determine at which of the stop points a mode change occurred, we make use of the unique characteristics of walk segments. As previous studies have shown (Chung and Shalaby, 2005; Tsui and Shalaby, 2006; Witayangkurn et al., 2013), on a fine scale the change of travel mode can be assumed always to be accompanied by a walk segment. Due to the fine-grained segmentation enabled by the high sampling rate, even if, for example, a person changes from bike to bus, there is a short walk segment in between, which helps to detect the mode change.

Walk segments can be easily detected due to their consistently low speeds (see Section 'Input variables' for details on speed-related variables). To avoid undetected mode changes, we 
propose not to use a minimum duration for walk segments. As suggested by Witayangkurn et al. (2013), mode-change points are detected by searching for the following patterns within consecutive segments:

- non-walk - stop - walk

- walk - stop - non-walk

Whenever a stop-point segment that has not been classified as walk (a non-walk segment) is followed by a walk segment, the stop point in between is declared a mode-change point. These mode-change points are the basis for the identification of single-mode segments - i.e. the second, higher-level segmentation stage. Single-mode segments are subsets of trajectories with distinct travel modes. They occur between two mode-change points and comprise one or more stop-point segments, as illustrated in Figure 2. Duration, length and speed-related indicators as well as aggregated heart rates are derived for single-mode segments as input variables for travel-mode detection, as described in the following sections.

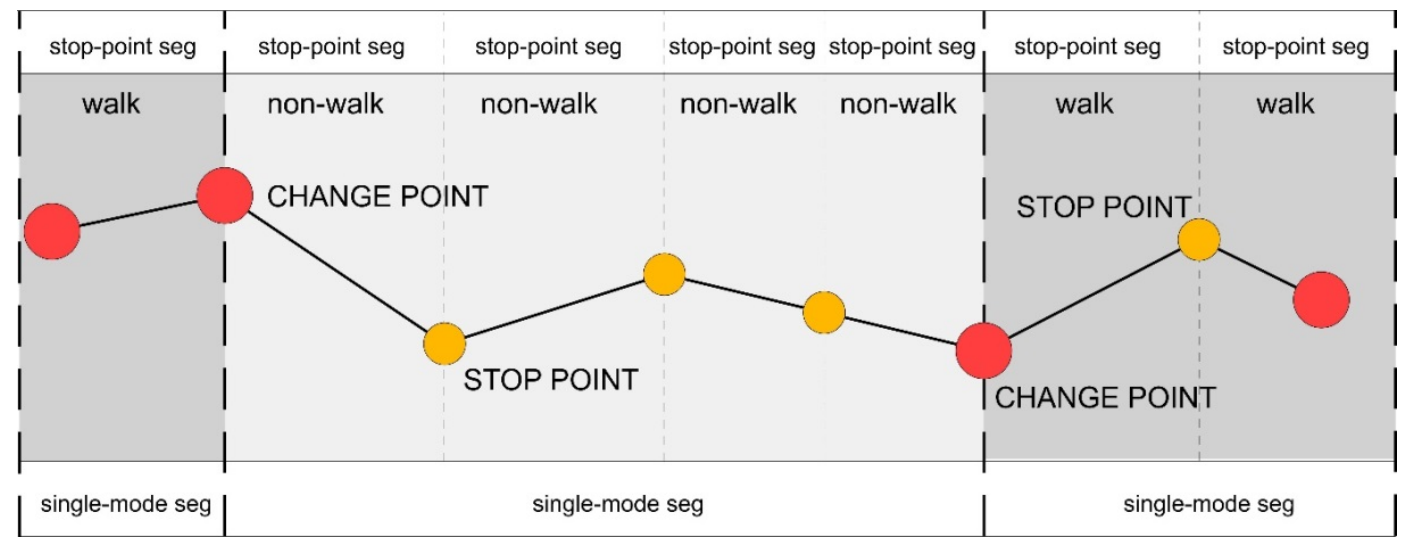

Figure 2: Schematic illustration of the two segmentation levels

\section{Rule-based Travel-Mode Detection (TMD)}

The proposed approach relies solely on the combination of speed-related variables, heart rates, and variables derived from the two-stage segmentation. The input variables, the iterative ruleset development and the classification procedure are described in more detail below.

\section{Input variables}

\section{Speed-related variables}

A basic but important variable for travel-mode detection is speed. As suggested by Stopher et al. (2008), our method uses the 85th percentile of speed per segment to identify a mode, as percentiles help to remove outliers caused by inaccurate GNSS measurements. Additionally, the standard deviation of speed within a single-mode segment is used, as it is expected to be considerably higher for motorized modes than for active modes. 


\section{Variables derived from two-stage segmentation}

Information about stop patterns can be derived from the two segmentation stages and is especially valuable for the distinction of motorized transport modes. An overview on segment-derived variables is provided in Table 1. In general, these segmentation-based variables allow a fine tuning of rulesets to regional characteristics, such as the distance between bus stops. However, in turn this limits the transferability of the rulesets.

\section{Heart rate variables}

Heart rate is a well-suited indicator for the intensity of physical activities, but due to interindividual differences comparing raw heart rates may not be meaningful. In anonymized data, detailed information on gender, age and measured resting heart rates for individual calibration is not available. As an approximated value for the resting heart rate, we propose to use the 0.1 st percentile of the heart rate per person. The raw heart-rate values in beats per minute are converted into percentages over the resting heart rate of the person concerned. Then for each single-mode segment, the 85th percentile of percentages over resting heart rate is derived.

Table 1: Input variablesfor Travel-Mode Detection based on single-mode segments

\begin{tabular}{|l|l|l|}
\hline Speed variables & Segment-derived variables & Heart rate variables \\
\hline $\begin{array}{l}\text { 85 } \\
\text { single-mode segment }\end{array}$ & $\begin{array}{l}\text { Median stop-point segment } \\
\text { duration* } \\
\text { per single-mode segment }\end{array}$ & $\begin{array}{l}\text { 85th percentile of percentage } \\
\text { over resting pulse per single- } \\
\text { mode segment }\end{array}$ \\
& $\begin{array}{l}\text { Median stop-point segment } \\
\text { length* }\end{array}$ & $\begin{array}{l}\text { Mediant stop-point duration* } \\
\text { Single-mode segment length } / \\
\text { count of stop points }\end{array}$ \\
& $\begin{array}{l}* \text { calculated per single-mode } \\
\text { segment }\end{array}$ & \\
\hline
\end{tabular}

\section{Iterative ruleset development}

Based on the input variables discussed above, each travel mode is described by one or several rules. The specific rule thresholds have to be calibrated based on the relevant dataset and adjusted in an iterative process of frequent plausibility checks. When visualizing random trajectories in a GIS environment, an expert familiar with the study area can easily identify the travel mode being undertaken, for example based on the routes taken. These visual 
checks and the adjustment of rulesets form an iterative process to improve the classification stepwise (see Figure 1).

It may occur that different types within one mode can be distinguished. For example, regional and city buses may have different speed and stop characteristics. In this case, it is preferable to differentiate these types in separate rulesets rather than to set generous thresholds which fit both types. Depending on the specific application, the different variables may be more or less conclusive for different travel modes. We propose using specific value ranges for the 85th speed percentiles and speed standard deviations for every mode. Segment-derived variables such as stop patterns are used for the distinction of motorized modes. Heart rates are expected to be especially valuable for differentiating between active and motorized modes. For active modes, we propose setting a threshold for the minimum heart rate in percentage over resting heart rate. However, we do not recommend determining a maximum value for heart rates in motorized modes, as for these modes speed is the more conclusive indicator.

\section{Hierarchical classification procedure}

Depending on the characteristics of the dataset at hand, the classification of some travel modes may perform better than others. Based on visual inspections, the expert who performs the classification can judge the reliability of the classification of the different modes and introduce a classification hierarchy by ranking the modes accordingly. If a segment matches several rulesets, the travel mode with the higher rank in the hierarchy is favoured and assigned. If a segment does not comply with any of the rulesets, it will remain unclassified.

Heart rates are an important additional indicator for the detection of active modes. This is expected to increase the reliability of the classification of active modes, giving them a higher rank in the classification hierarchy. Due to the hierarchy of modes, the classifications of different modes are interdependent. Thus heart rate improves the classification not only of active modes, but also of motorized ones.

\section{Case Study}

This section describes a case study in which the proposed method was successfully applied in the region of Salzburg, Austria. To assess the added value of the heart-rate data, the classification was conducted with and without heart rates. The analysis was performed in a PostgreSQL/PostGIS database using Structured Query Language (SQL). For visual inspection and plausibility checks, we used the software packages QGIS and Tableau Desktop. Adjusting the rulesets in our SQL scripts and frequent visual checks formed an iterative process. The description of specific rulesets and value ranges as defined in the case study is provided in the Supplementary Material section. However, it should be noted that rulesets and parameterization may not be transferable and that they always need to be calibrated based on the dataset at hand. 


\section{Data}

The data used for this case study was collected in the context of the GISMO research project (Geographical Information Support for Healthy Mobility) in Salzburg, Austria, led by the Department of Geoinformatics, Z_GIS, University of Salzburg. One of the aims of the project is to investigate the health effects of switching from car to active commuting, stimulated by measures in the context of corporate mobility management. Within the GISMO project, 70 subjects from a randomized, clinical intervention study are required to document their commuting trips in a travel diary and wear fitness watches (Polar ${ }^{\circledR}$ M200) for 2 weeks at the beginning and end of the one-year intervention period. The raw data from the first two weeks comprised 2.17 million location and heart rate records, belonging to 1,007 trajectories recorded at a regular sampling rate of one second.

\section{Data Preprocessing}

First, we removed (a) trajectories without location information; (b) trajectories that were short in terms of either distance or time; (c) records at the beginning or end of a trajectory without any location information; (d) trajectories without any significant movement. After data cleansing, about $74 \%$ of the original data records were used for further analysis - that is, about 1.6 million records for 810 trajectories. The speed for each record was calculated using the distance from the previous record. For the detection of speed outliers, we used variables such as the speed z-score per trajectory, and adjusted the thresholds based on frequent checks with the dataset. The outliers identified were interpolated using the average of the 3 preceding and 3 following records, while excluding the outliers themselves. For more details on speed-correction for outliers, see the Supplementary Material. Even though the speed values were interpolated in less than $2 \%$ of all records, speed diagrams showed that the quality of the speed values was increased substantially. It should be noted that the interpolation only affects the speed values; location inaccuracies are not corrected.

\section{Segmentation and travel-mode detection}

The two stages of segmentation were performed, as described in Section 'Two-stage Trajectory Segmentation'. On average, a trajectory had 1.9 change points, which seems plausible assuming that only walk segments do not have any mode-change points. Details on the detection of each mode are outlined below.

\section{Motorized travel modes}

As characteristics such as the speed ranges of motorized modes differ in urban and rural contexts, subcategories were introduced. While car segments within the city boundaries were characterized by lower speed thresholds, those from highways or motorways were detected using a ruleset with higher speed thresholds. For cars, the stop-point duration was of special interest, as it was found that cars tended to stop for a shorter time than buses or trains.

The different characteristics between city and regional buses also required the development of two different rulesets, while the train mode was split into three categories: local train, regional train and high-speed train. For the bus and train rulesets, besides speed variables, 
segment-derived variables such as duration and length between stop points, total number of stop points and duration of stop points were especially useful.

\section{Active travel modes}

Heart rates are a valuable additional indicator to prevent confusion of active and motorized modes. But as the walk mode clearly differs from other modes due to its considerably lower speeds, it is not necessary to include heart rates for walk-mode detection. Therefore, heart rates were only included in the bike rulesets, in addition to value ranges for the 85th speed percentile. For bike mode, we distinguished two subcategories: slower cycling with lower heart rates, and faster cycling with higher heart rates.

The reliability hierarchy of the modes (from low to high reliability) was found to be: car train - bus - bike - walk.

The travel-mode detection was performed without and with heart rates, in order to determine the added-value of considering heart rates. Figure 3 provides an example of different classifications for the two versions. In this example, a person walks from the start point to a bus stop, takes the bus to the station, and then changes to the train. In the classification without heart rates, the bus segment is falsely classified as bike. In the classification with heart rates, the ruleset for bike mode includes a minimum threshold for bike classification of $57 \%$ over resting heart rate. With $30 \%$ over the resting level, the segment does not meet this threshold. As a consequence, it is classified correctly as bus.
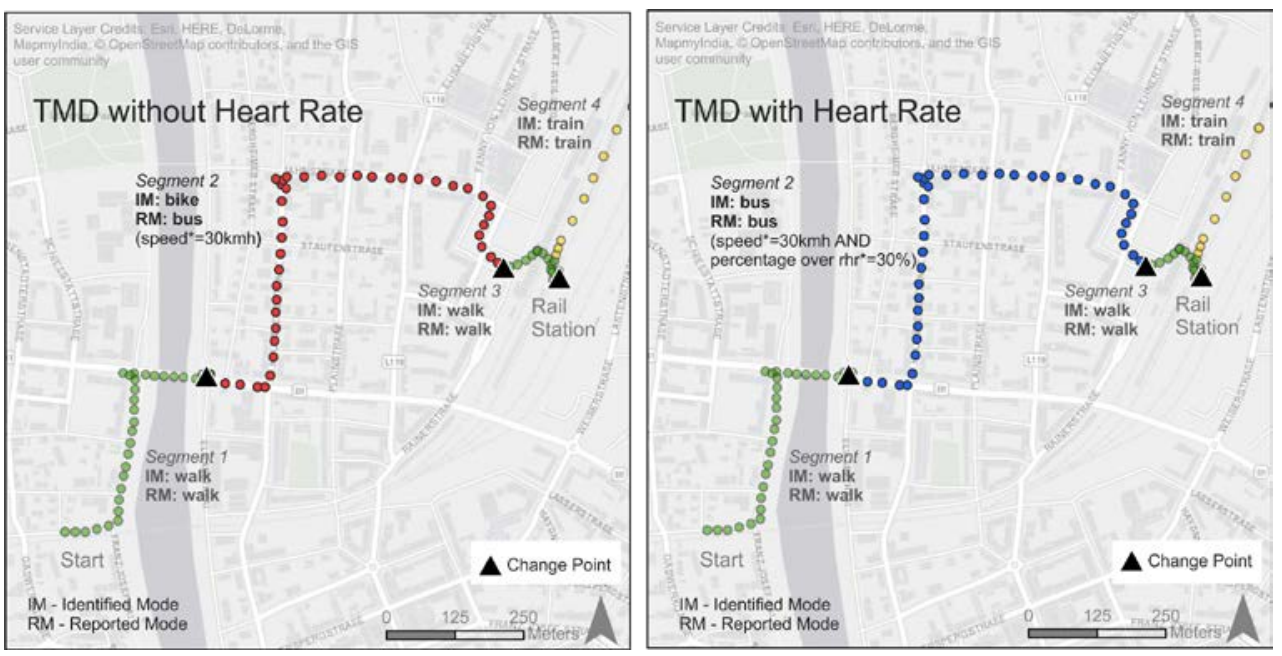

Figure 3: Example of a trajectory with bus segment falsely classified as bike in the TMD without heart rates (left), and correctly classified as bus segment in the TMD with heart rates (right)

speed* - 85th percentile of speed

percentage over hin - 85th percentile of percentage over resting heart rate 


\section{Validation}

The application of the proposed method was validated with ground truth data from study participants' travel diaries. Out of 2,209 classified segments, the validation was performed for 409 segments where complete ground truth was available. As, in the diaries, bus and train modes were not reported individually but subsumed as public transport, these modes were also taken together for validation. The validation results for the method both without and with heart rates are provided in Tables 2 and 3.

The Producer's accuracy $(\mathrm{Pa})$ represents the share of correctly identified segments. The very high rate of $98.3 \%$ correctly classified bike segments even without heart rates is striking. But this can be explained by an overclassification due to the higher weight that was given to the bike mode in the hierarchical classification process: if a segment matches several modes, e.g. bike and bus, it is always classified as bike. While this means that almost all bike segments are correctly identified as bike (high $\mathrm{Pa}$ ), it also leads to a high number of segments that are classified as bike while actually being motorized modes (lower User's accuracy (Ua) of 87.4\% for bike). This error can be reduced substantially by including heart rates in the classification. The user's accuracy for bike mode is improved by 10.7 percentage points (pp), from $87.4 \%$ to $98.1 \%$. Likewise the Pas for motorized modes are substantially improved when heart rates are included, by $11.1 \mathrm{pp}$ for car mode, and $17.7 \mathrm{pp}$ for public transport; for the bike and walk Pas and the total accuracy $(\mathrm{Ta})$, the heart rate does not make a big difference. In other words, the number of falsely classified bike segments is clearly reduced, while the number of correctly classified motorized mode segments is clearly increased. In summary, heart rates proved to be very valuable for differentiating motorized and active modes.

Table 2: Confusion matrix for classified segments without consideration of heart rates

\begin{tabular}{|c|c|c|c|c|c|c|c|c|}
\hline & \multicolumn{7}{|c|}{$\begin{array}{l}\text { IDENTIFIED MODES } \\
\text { WITHOUT CONSIDERATION OF HEART-RATE }\end{array}$} \\
\hline & & Walk & Bike & $\begin{array}{l}\text { Public } \\
\text { Transport }\end{array}$ & Car & $\begin{array}{c}\text { Un- } \\
\text { classified }\end{array}$ & Total & $\mathrm{Pa}(\%)$ \\
\hline \multirow{6}{*}{ 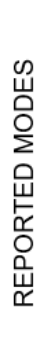 } & Walk & 80 & 2 & 0 & 0 & 2 & 84 & $95.2 \%$ \\
\hline & Bike & 1 & 212 & 0 & 2 & 1 & 219 & $98.2 \%$ \\
\hline & $\begin{array}{l}\text { Public } \\
\text { Transport }\end{array}$ & 4 & 20 & 19 & 33 & 3 & 79 & $24.1 \%$ \\
\hline & Car & 0 & 9 & 4 & 14 & 0 & 27 & $51.9 \%$ \\
\hline & Total & 85 & 246 & 23 & 49 & 6 & 409 & \\
\hline & Ua (\%) & $94.1 \%$ & $87.4 \%$ & $82.6 \%$ & $67.3 \%$ & & \multicolumn{2}{|c|}{$\mathrm{Ta}=80.2 \%$} \\
\hline
\end{tabular}


Table 3: Confusion matrix and differences in accuracies for classified segments with consideration of heart-rates

\begin{tabular}{|c|c|c|c|c|c|c|c|c|c|}
\hline & \multicolumn{7}{|c|}{$\begin{array}{c}\text { IDENTIFIED MODES } \\
\text { WITH CONSIDERATION HEART-RATE }\end{array}$} & \multirow[b]{2}{*}{$\begin{array}{l}\text { Diff. } \\
\text { (pp) }\end{array}$} \\
\hline & & Walk & Bike & $\begin{array}{l}\text { Public } \\
\text { Transport }\end{array}$ & Car & $\begin{array}{l}\text { Un- } \\
\text { classified }\end{array}$ & Total & $\mathrm{Pa}(\%)$ & \\
\hline \multirow{7}{*}{ 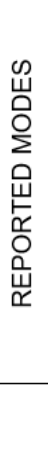 } & Walk & 80 & 1 & 0 & 0 & 3 & 84 & $95.2 \%$ & 0.0 \\
\hline & Bike & 1 & 212 & 0 & 0 & 6 & 219 & $96.8 \%$ & -2.3 \\
\hline & $\begin{array}{l}\text { Public } \\
\text { Transport }\end{array}$ & 0 & 3 & 33 & 31 & 12 & 79 & $41.8 \%$ & 17.7 \\
\hline & Car & 0 & 0 & 6 & 17 & 4 & 27 & $63.0 \%$ & 11.1 \\
\hline & Total & 81 & 216 & 39 & 48 & 25 & 409 & & \\
\hline & Ua (\%) & $98.8 \%$ & $98.1 \%$ & $84.6 \%$ & $64.6 \%$ & & \multicolumn{2}{|c|}{$T a=83.6 \%$} & \\
\hline & $\begin{array}{l}\text { Diff. } \\
\text { (pp) }\end{array}$ & 4.7 & 10.7 & 2.0 & -2.7 & & & & \\
\hline
\end{tabular}

In those segments which were correctly classified, heart rate percentages over resting level for walk, bike and motorized modes vary significantly (see Figure 4). The clearly higher heart-rate levels for bike mode are striking and confirm that a minimum heart-rate threshold for bike mode is highly useful. In contrast, walk mode exhibits a higher dispersion towards lower heart-rate levels, which confirms that a threshold for heart rate for walk mode is not advisable.

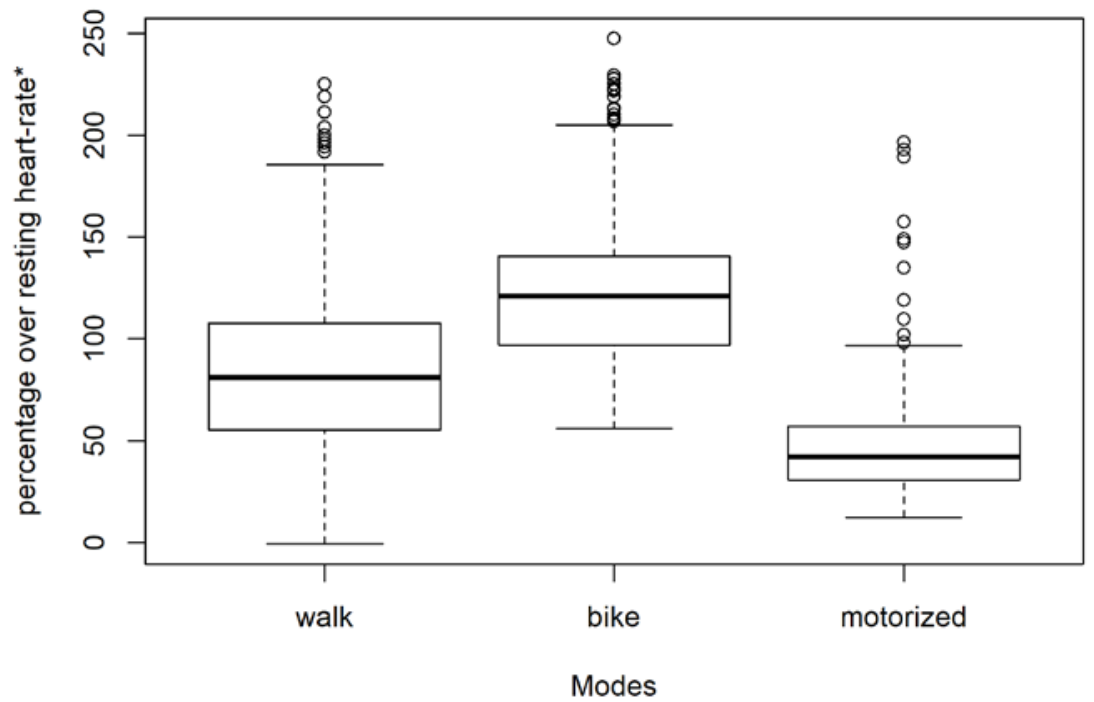

Figure 4: Boxplots of heart-rate percentages over resting level for walk, bike and motorized modes in the correctly classified segments

* based on 85 percentile heart rate per single-mode segment 


\section{$5 \quad$ Results and Discussion}

This paper presents a rule-based method for travel-mode detection for data from off-theshelf fitness watches based exclusively on speed, heart rate and derived features, making equipment requirements minimal. Heart rates are calibrated based on an approximated resting pulse per person, which makes the method suitable for anonymized data without individual-based information on gender, age or fitness levels. In our case study, the method performed very well and substantially improved the differentiation between active and motorized modes compared to detection that did not consider heart rates. Walk segments could be classified very accurately based on their low speed. But heart rates proved to be a highly useful additional indicator to reliably distinguish bike from motorized modes.

We showed that heart rates in combination with GNSS data are very useful for travel-mode detection. This combination allows a considerable reduction in the number of input variables, and a substantial scaling-down of the processing effort, especially as prior mapmatching can be omitted.

One of the limitations of the proposed method is its need for laborious calibration to the dataset of interest. Once calibrated, the rulesets for motorized modes in particular, which depend on stop patterns, are tailored to regional characteristics and therefore cannot be transferred to other datasets. In the future, the differentiation among motorized modes as well as the transferability of the method could be further improved by including additional variables such as acceleration, heading and GIS data. The method was designed for detection of the travel modes walk, bike, bus, train and car, but further travel modes could also be considered.

The growing amount of data from location-aware activity trackers is a valuable data source for mobility research, particularly for the detection of intermodal trips. Our method makes use of these new data sources and takes into account the potential of physiological indicators for automatic travel-mode detection.

\section{Supplementary Material}

A detailed description of the rulesets and value ranges as used in the case study is provided at: https://petrastutz.wordpress.com/2018/06/18/workflow-for-travel-mode-detection/ 


\section{Acknowledgements}

The Austrian Ministry of Transport, Innovation and Technology (BMVIT) funded parts of this work in the context of the research project GISMO (FFG Nr. 854974). The project is coordinated by the Department of Geoinformatics, Z_GIS, University of Salzburg. Other partners are: RSA Studio iSpace; University Hospital Zurich - Department of Cardiology; University Hospital Salzburg - Department of Sport Medicine; TraffiCon, and Herry Consult. The case study data was collected and kindly provided by the University Hospital Salzburg (SALK).

We appreciate the helpful feedback of the three anonymous reviewers.

\section{References}

Bohte, W., \& Maat, K. (2009). Deriving and validating trip purposes and travel modes for multi-day GPS-based travel surveys: A large-scale application in the Netherlands. Transportation Research Part C: Emerging Technologies, 17(3), 285-297.

Brage, S., Ekelund, U., Brage, N., Hennings, M. A., Froberg, K., Franks, P. W., \& Wareham, N. J. (2007). Hierarchy of individual calibration levels for heart rate and accelerometry to measure physical activity. Journal of Applied Physiology, 103(2), 682-692. https://doi.org/10.1152/japplphysiol.00092.2006

Chung, E.-H., \& Shalaby, A. (2005). A Trip Reconstruction Tool for GPS-based Personal Travel Surveys. Transportation Planning and Technology, 28(5), 381-401. https://doi.org/10.1080/03081060500322599

Costa, S., Ogilvie, D., Dalton, A., Westgate, K., Brage, S., \& Panter, J. (2015). Quantifying the physical activity energy expenditure of commuters using a combination of global positioning system and combined heart rate and movement sensors. Preventive Medicine, 81(Supplement C), 339-344. https://doi.org/10.1016/j.ypmed.2015.09.022

Gong, H., Chen, C., Bialostozky, E., \& T. Lawson, C. (2012). A GPS/GIS method for travel mode detection in New York City. Computers, Environment and Urban Systems, 36. https://doi.org/10.1016/j.compenvurbsys.2011.05.003

Gong, L., Morikawa, T., Yamamoto, T., \& Sato, H. (2014). Deriving Personal Trip Data from GPS Data: A Literature Review on the Existing Methodologies. Procedia - Social and Behavioral Sciences, 138, 557-565. https://doi.org/10.1016/j.sbspro.2014.07.239

James, P., Weissman, J., Wolf, J., Mumford, K., Contant, C. K., Hwang, W. T., ... \& Glanz, K. (2016). Comparing GPS, log, survey, and accelerometry to measure physical activity. American journal of health behavior, 40(1), 123-131.

Nitsche, P., Widhalm, P., Breuss, S., Brändle, N., \& Maurer, P. (2014). Supporting large-scale travel surveys with smartphones-A practical approach. Transportation Research Part C: Emerging Technologies, 43, 212-221.

Ogilvie, D., Panter, J., Guell, C., Jones, A., Mackett, R., \& Griffin, S. (2016). Health impacts of the Cambridgeshire Guided Busway: a natural experimental study. Public Health Research, 4(1), 1-154. https://doi.org/10.3310/phr04010

Pärkkä, J., Ermes, M., Korpipaa, P., Mantyjarvi, J., Peltola, J., \& Korhonen, I. (2006). Activity classification using realistic data from wearable sensors. IEEE Transactions on Information Technology in Biomedicine, 10(1), 119-128. 
Quddus, M. A., Ochieng, W. Y., \& Noland, R. B. (2007). Current map-matching algorithms for transport applications: State-of-the art and future research directions. Transportation Research Part C: Emerging Technologies, 15(5), 312-328. https://doi.org/10.1016/j.trc.2007.05.002

Siła-Nowicka, K., Vandrol, J., Oshan, T., Long, J. A., Demšar, U., \& Fotheringham, A. S. (2016). Analysis of human mobility patterns from GPS trajectories and contextual information. International Journal of Geographical Information Science, 30(5), 881-906.

Stopher, P., Clifford, E., Zhang, J., \& FitzGerald, C. (2008). Deducing mode and purpose from GPS data. Institute of Transport and Logistics Studies, 1-13.

Tsui, S., \& Shalaby, A. (2006). Enhanced System for Link and Mode Identification for Personal Travel Surveys Based on Global Positioning Systems. Transportation Research Record: Journal of the Transportation Research Board, 1972, 38-45. https://doi.org/10.3141/1972-07

Witayangkurn, A., Horanont, T., Ono, N., Sekimoto, Y., \& Shibasaki, R. (2013). Trip reconstruction and transportation mode extraction on low data rate gps data from mobile phone. In Proceedings of the international conference on computers in urban planning and urban management (CUPUM 2013) (pp. 1-19). Retrieved from

http:// cupum2013.geo.uu.nl/download/usb/contents/pdf/shortpapers/53_Witayangkurn.pdf

Zhang, L., Dalyot, S., Eggert, D., \& Sester, M. (2011). Multi-stage approach to travel-mode segmentation and classification of gps traces. International Archives of the Photogrammetry, Remote Sensing and Spatial Information Sciences:[Geospatial Data Infrastructure: From Data Acquisition And Updating To Smarter Services] 38-4 (2011), Nr. W25, 38(W25), 87-93.

Zong, F., Yuan, Y., Liu, J., Bai, Y., \& He, Y. (2017). Identifying travel mode with GPS data. Transportation Planning and Technology, 40(2), 242-255.

https://doi.org/10.1080/03081060.2016.1266170 